East African Medical Journal Vol. 87 No. 5 May 2010

SELF-REPORTED ATTENTION DEFICIT AND HYPERACTIVITY DISORDER SYMPTOMS AMONG UNIVERSITY STUDENTS IN ELDORET, KENYA

L. Atwoli, MBChB, MMed Psych (UoN), Lecturer, P. Owiti, MBChB, G. Manguro, MBChB and D. Ndambuki, MBChB, Department of Mental Health, Moi University School of Medicine, P.O. Box 4606 - 30100, Eldoret, Kenya

Request for reprints to: Dr. L. Atwoli, Department of Mental Health, Moi University School of Medicine, P.O. Box 149330100, Eldoret, Kenya

\title{
SELF-REPORTED ATTENTION DEFICIT AND HYPERACTIVITY DISORDER SYMPTOMS AMONG UNIVERSITY STUDENTS IN ELDORET, KENYA
}

\author{
L. ATWOLI, P. OWITI, G. MANGURO and D. NDAMBUKI
}

\begin{abstract}
Objective: To determine the prevalence of self-reported attention deficit and hyperactivity disorder (ADHD) symptoms among university students in Eldoret, Kenya.

Design: A cross-sectional descriptive study of all students who gave consent to participate in the study.

Setting: Moi University's Town Campus, comprising the Schools of Medicine, Dentistry and Public Health.

Subjects: Four hundred and fifty eight undergraduate students, with a mean age of 23.7 years $(17-46$, S.D. 4.1$)$, of whom $236(51.5 \%)$ were male.

Main outcome measures: Presence of ADHD symptoms as measured by the adult ADHD self-report scale (ASRS versus 1.1).

Results: The prevalence rate of self-reported ADHD symptoms was $21.8 \%$. This was significantly associated with belonging to a younger age-group (17-20 years) compared to the older age-groups $(p<0.05)$. The prevalence was higher among females $(25.2 \%)$ than among males $(\mathbf{1 8 . 6 \%})$, but this was not statistically significant. There was no significant association between a positive ASRS screen and course of study. Using a modification of ASRS full symptom checklist to approximate a DSM IV TR ADHD diagnosis yielded a prevalence of ADHD symptoms of $9.2 \%$ in this cohort.

Conclusion: The prevalence rate of ADHD symptoms among university students in Eldoret, Kenya is significantly higher than that reported in other studies. Further studies are needed to generate more information about this condition in the lowincome countries, especially in college and university populations.
\end{abstract}

\section{INTRODUCTION}

The DSM-IV-TR (1) criteria for diagnosis of ADHD include the manifestation of at least six out of eighteen possible symptoms over a considerable period of time (at least six months). These can be wholly symptoms of inattention (inattentive subtype) or hyperactivity-impulsivity symptoms (hyperactiveimpulsive subtype), while the combined subtype requires that both criteria for the inattentive and the hyperactivity / impulsive subtypes be met during the same six-month period (1).

In population based studies, the prevalence rate of ADHD among children and adolescents has ranged from $1-7.3 \%$ with wide variations reported in many studies (2-5). Some authorities estimate the worldwide pooled prevalence of ADHD to be $5.29 \%$ $(2,6)$.

As opposed to childhood ADHD, only recently has adult ADHD become a focus of widespread clinical attention $(7,8)$. Most of the previous epidemiological studies did not include adults in their samples (5). In fact treatment was sometimes formally stopped at adolescence (9). Although the symptoms of ADHD have been reported to decline during adolescence, with a few studies reporting that they almost become negligible or compensated in adulthood (8), in a number of patients the symptoms often persist into adulthood $(4,5,9,10)$.

The estimated adult prevalence rate currently is at between 3-5\% but there have been wide variations across studies $(4,5,10-12)$. It has therefore been thought that most symptoms remit in adulthood although evidence now suggests that a contributing factor to the perceived decreased prevalence could be the fact that the DSM-IV-TR diagnostic criteria was formulated with children in mind and adults only 'outgrow' the diagnostic criteria $(2,5)$.

Though most of those with ADHD never achieve their full academic potentials, even those 
who have gained entry to the universities have not been spared of the condition. Given the academic under-achievement, some writers have predicted a lowered prevalence rate in the university setting and have put it at between 1-3\% (3). However, a study carried out in several universities in three countries (Italy, New Zealand and the United States) found a prevalence of between $0-8.1 \%$ (3) with the mode below that of the general population. Though slightly modest as compared to the general adult population this problem is still significant enough (13). Another screening study found a prevalence of $7 \%$ of significant ADHD symptoms among college students (14).

There is a paucity of studies on ADHD from low-income countries in general and from Africa in particular, and this study aimed to broaden the body of knowledge in this field by generating new knowledge on ADHD among Kenyan university students.

\section{MATERIALS AND METHODS}

Site: The study was carried out at the Town Campus of Moi University. The campus is located in Eldoret town, about $320 \mathrm{~km}$ North West of the capital Nairobi, Kenya. The campus has three schools:

(i) The School of Medicine offering undergraduate courses in Bachelor of Medicine and Bachelor of Surgery (MBChB) with 556 students and Bachelor of Science in Nursing (BSc. Nur) with 135 students;

(ii) The School of Public Health offering an undergraduate degree in Environmental Health (BSc. Env. Health) with 208 students, and

(iii) TheSchool of Dentistry offering anundergraduate course in Dental Health (BDS) with 37 students.

Participants: The study aimed at recruiting all the students at the Moi University town campus (936 students at the time of the study). A total of 507 students were available and accepted to participate in the study, and out of these $458(90.3 \%)$ returned questionnaires that were correctly filled. Slightly over half of the participants were male $(51.5 \%)$. Majority of the participants were medical students (55.2\%) while the others were environmental health students $(26.0 \%)$, Nursing students $(12.7 \%)$ and dental students (6.1\%). Table 1 shows the distribution of the participants by course and year of study.

Design: This was a cross-sectional descriptive survey using the Adult ADHD self-report scale (ASRS v1.1) to determine the prevalence of self-reported ADHD symptoms among university students in Eldoret, Kenya. The ASRS (15) is a self-administered questionnaire developed by the World Health Organisation to be used as a screening tool for adult ADHD. It consists of 18 questions derived from the DSM IV TR diagnostic criteria for ADHD and is divided into two parts. Part A consists of a six question screener that has been validated elsewhere as a sensitive screen for adult ADHD. Part B consists of the remaining items on the DSM IV TR ADHD diagnostic criteria. The first three questions on the screener require a response in the range of 'sometimes' to 'very often' to be considered positive while the remaining three require an 'often' or 'very often' response. Four positive responses in part A constitute a positive screen for adult ADHD. PartB is not required for purposes of screening for adult ADHD.

In this study, an attempt was also made to come up with an approximation of the DSM IV TR diagnostic criteria for ADHD using the ASRS symptom checklist. A modification was introduced requiring endorsement of an item as 'often' or 'very often' for a positive score. DSM IV TR (1) diagnostic criterion A for ADHD was then used to screen for possible ADHD in the participants. To meet the criteria for possible ADHD, at least six of either the inattentive symptoms (Questions 1-4 and 7-11 on the ASRS checklist) or the hyperactivity/impulsivity symptoms (Questions 5, 6 and 12-18 on the ASRS checklist) had to be positive on the full ASRS symptom checklist.

A brief questionnaire for collection of sociodemographic datawas alsoused. Datacollectedincluded date of birth, gender, course and year of study.

Procedure: The study was carried out in the period March to October, 2009. Prior to data collection, ethical review was done by the Institutional Research and Ethics Committee (IREC) of Moi University and Moi Teaching and Referral Hospital. Questionnaires were distributed according to the known number of students in each class. Class representatives distributed the questionnaires to their classmates prior to the start of whole-class activities and collected them at the end of the class. Each questionnaire had a detachable section with a unique number linked to the full questionnaire and known only to each individual participant for the purposes of feedback.

Data storage and analysis: Collected data was entered into a Microsoft Access database and analysed using SPSS version 12.0. Descriptive statistics were used to compute means and standard deviations for numerical variables as well as frequencies for nominal and ordinal variables. The Chi square test statistic $\left(\mathrm{X}^{2}\right)$ was used to test the significance of association between various factors and a positive ASRS screen. A value of $\mathrm{p}<0.05$ was considered statistically significant.

Feedback: Every participant received feedback on the screen result and its implication, and those with positive screens were advised to seek a consultation with a mental health specialist. The feedback report was only linked to the unique number on each questionnaire, and the results were sealed and delivered through the respective class representatives. 


\section{RESULTS}

Description of the participants: The study involved 458 participants with a mean age of 23.7 years (17-46, S.D. 4.1). Sixty nine (15.1\%) of the participants were aged 17-20 years, $249(54.4 \%)$ were aged 21-24 years and $140(30.6 \%)$ were 25 years and older. In this study, $51.5 \%$ of the participants were male and $32.1 \%$ were in their first year of study in the various courses. Table 1 shows the distribution of the participants by course and year of study.

Only one participant indicated that he had been earlier diagnosed with ADHD and was on treatment at the time of the study.
Self-reported ADHD symptoms: In this study, the prevalence of self-reported ADHD symptoms was $21.8 \%$. Table 2 shows the distribution of selfreported ADHD symptoms with various variables. The only variable showing significant association with ADHD symptoms was younger age-group. Using the modification described in the methods section to approximate a DSM IV TR diagnosis, 42 $(9.2 \%)$ met the criteria for possible ADHD. A larger proportion of those meeting these criteria had the hyperactive/impulsive type of symptoms. Table 3 shows the distribution of symptoms by type of ADHD.

Table 1

Distribution of respondents by course and year of study

\begin{tabular}{lllllllllllll}
\hline & \multicolumn{1}{c}{} & \multicolumn{1}{c}{ Course } \\
& \multicolumn{1}{c}{ Medicine $^{\mathrm{a}}$} & \multicolumn{1}{c}{ Nursing } & \multicolumn{2}{c}{ Env. Health } & \multicolumn{2}{c}{ Dentistry $^{\mathrm{c}}$} & \multicolumn{2}{c}{ Total } \\
& No. & $(\%)$ & No. & $(\%)$ & No. & $(\%)$ & No. & $(\%)$ & No. & $(\%)$ \\
\hline Year of study & 1 & 57 & 22.5 & 12 & 20.7 & 60 & 50.4 & 18 & 64.3 & 147 & 32.1 \\
& 2 & 25 & 9.9 & 15 & 25.9 & 13 & 10.9 & 10 & 35.7 & 63 & 13.8 \\
& 3 & 33 & 13.0 & 4 & 6.9 & 28 & 23.5 & - & - & 65 & 14.2 \\
& 4 & 29 & 11.5 & 27 & 46.6 & 18 & 15.1 & - & - & 74 & 16.2 \\
& 5 & 56 & 22.1 & - & - & - & - & - & - & 56 & 12.2 \\
& 6 & 53 & 20.9 & - & - & - & - & - & - & 53 & 11.6 \\
\hline Total & 253 & 100 & 58 & 100 & 119 & 100 & 28 & 100 & 458 & 100 \\
\hline \% of Total $(\mathrm{n}=458)$ & & 55.2 & & 12.7 & & 26.0 & & 6.1 & & 100 \\
\hline
\end{tabular}

${ }^{a}$ Six-year course ${ }^{b}$ Four-year course ${ }^{c}$ Five-year course with only the first two years

Table 2

Variation of ADHD with socio-demographic factors

\begin{tabular}{lrll}
\hline Variable (No.) & ADHD $(\%) X^{2}$ & P-value \\
\hline Age-group & & & \\
17-20 (n=69) & 30.4 & 10.636 & $0.005^{*}$ \\
$21-24(\mathrm{n}=249)$ & 24.5 & & \\
25 and older (n=140) & 12.9 & & \\
Gender & & & \\
Female (n=222) & 25.2 & 2.903 & 0.091 \\
Male (n=236) & 18.6 & & \\
Course & & & \\
Dentistry (n=28) & 32.1 & 4.713 & 0.194 \\
Medicine (n=253) & 23.7 & & \\
Nursing (n=58) & 20.7 & & \\
Env. Health (n=119) & 16.0 & & \\
\hline
\end{tabular}

*Statistically significant
Table 3

Types of $A D H D$

\begin{tabular}{lcc}
\hline & \multicolumn{2}{c}{ Frequency } \\
Type & No. & $(\%)$ \\
\hline Inattentive & 15 & 35.7 \\
Hyperactive/Impulsive & 17 & 40.5 \\
Combined & 10 & 23.8 \\
\hline Total & 42 & 100 \\
\hline
\end{tabular}

\section{DISCUSSION}

This is one among very few ADHD studies involving university students in a low-income country, and it will therefore serve to broaden the body of knowledge on ADHD in this setting. The self-reported ADHD symptom prevalence rate of $21.8 \%$ washigher than any comparable studies in other settings. This raises the possibility that this instrument, which has never been used in this setting before, may beover-inclusive with 
too many false positives. Indeed, many researchers have attributed the wide variation in prevalence of ADHD to the different methodologies used in the studies, rather than to any cultural or geographical difference between the populations $(5,6)$.

Another possibility is that ADHD rates are actually higher in this setting than in western settings. This would indicate that community prevalence rates are even higher than that suggested by this study, as demonstrated by studies that have shown that ADHD prevalence rates are lower in university and college populations than in the general population $(3,13,14)$. Populationbased studies using the same instrument suitably modified and validated for this population would be necessary to confirm this trend.

In this cohort, ADHD symptoms were higher among participants aged 17-20 years compared with older participants. This is consistent with findings in other studies, especially in Western settings that suggest an age-dependent decline in ADHD symptoms (8). The possibility of the more severely ill students having difficulties progressing with their studies cannot be ruled out as a cause of this apparent decline in symptoms with age.

The lack of significant difference between the genders seems to agree with Simon et al (2) in their meta-analysis that demonstrated that rates of adult ADHD do not vary with gender. Studies among children have consistently demonstrated gender differences, but these are eroded in older populations (4).

The finding that only one participant in this study had been diagnosed and was on treatment for ADHD suggests the possibility that ADHD is inadequately diagnosed and treated in this population. Studies elsewhere have also found that adult ADHD is still under-recognised and when treated it is not for the ADHD but for the emotional, behavioural and other comorbidities they present with, including antisocial personality, mood, anxiety, and substance use disorders $(4,5,7,16)$.

The high rates of self-reported ADHD symptoms in this study demonstrate the need for clinicians to have a high index of suspicion especially when confronted with students having academic and interpersonal difficulties.

In this study, apart from using the ASRS screener to identify students with possible ADHD, the researchers also attempted an approximation of the DSM IV TR ADHD criteria A symptoms using the full ASRS instrument. A lower rate of 9.2\% was found by using a stricter though untested criteria that required all the symptoms to meet the 'often' symptom threshold for a positive score.

The resulting symptom rate of $9.2 \%$ is still high compared to the rates under $7.3 \%$ reported from other studies (1-5). This suggests the need for more epidemiological studies in the developing world and especially among older populations in order to establish baseline rates.

Using the modification described above, symptoms of the hyperactive-impulsive type of ADHD were found to be more prevalent $(40.5 \%)$ compared to the inattentive type $(35.7 \%)$ and the combined type $(23.8 \%)$. In contrast, Heiligenstein et al (13) found a majority (56\%) of students meeting criteria for the inattentive type and the remainder equally distributed between the hyperactiveimpulsive and combined types (22\% each). This difference may be attributed to the variation in methodology and instruments used, but there is also a possibility that the differences are real and need further investigation.

A major limitation of this study was the lack of a confirmatory clinical examination for even a sample of the population. This means that these findings only relate to self-reported symptoms of ADHD and not the syndrome itself. Despite the fact that validation of the screener yielded a high correlation with clinical examination in comparison with clinical diagnoses $(15,17)$, the screener itself is not a diagnostic tool and anyone reporting threshold symptoms would require evaluation by a mental health specialist.

In conclusion, this study has demonstrated a high prevalence rate of self-reported ADHD symptoms in a population of university students in a lowincome country. Apart from a decline of symptoms with increasing age, no other socio-demographic factors were shown to be significantly associated with ADHD symptomatology. Further studies are needed to confirm these findings and to investigate any association between ADHD and other mental disorders among other variables.

\section{ACKNOWLEDGEMENT}

To all the students of Moi University Town campus who agreed to participate in this study.

\section{REFERENCES}

1. American Psychiatric Association. Diagnostic and Statistical Manual of Mental Disorders, $4^{\text {th }} \mathrm{ed}$, Text Revision. American Psychiatric Association, 2000.

2. Simon, V., Czobor, P., Balint, S., Meszaros, A. and Bitter, I. Prevalence and correlates of adult attentiondeficit hyperactivity disorder: meta-analysis. Brit. J. Psych. 2009; 194: 204-211.

3. DuPaul, G.J., Schaughency, E.A., Weyandt, L.L., et al. Self-report of ADHD symptoms in university students: Cross-gender and cross-national prevalence. J. Learn Disabil. 2001; 34: 370-379.

4. Montes, L.G.A., Garda, A.O.H. and RicardoGarcell, J. ADHD prevalence in adult outpatients with nonpsychotic psychiatric Illnesses. J. Atten. Disord. 2007; 11: 150-156. 
5. Fayyad,J., DeGraaf, R., Kessler, R., et al. Cross-national prevalence and correlates of adult attention-deficit hyperactivity disorder. Brit. J. Psych. 2007; 190: 402409.

6. Polanczyk, G., Silva de Lima, M., Horta, B.L., Biederman, J. and Rohde, L.A. The worldwide prevalence of ADHD: A systematic review and metaregression analysis. Am. J. Psych. 2007; 164: 942-948.

7. Kessler, R.C., Adler, L., Barkley, R., et al. The prevalence and correlates of adult ADHD in the United States: Results from the national co morbidity survey replication. Am. J. Psych. 2006; 163: 716-723.

8. Hill,J.C. and Schoener, E.P.Age-Dependent decline of attention deficit hyperactivity disorder. Am. J. Psych. 1996; 153:1143-1146.

9. McCarthy, S., Asherson, P., Coghill, D., et al. Attention-deficit hyperactivity disorder: treatment discontinuation in adolescents and young adults. Brit. J. Psych. 2009; 194: 273-277.

10. Kolar, D., Keller, A., Golfinopoulos, M., et al. Treatment of adults with attention-deficit/ hyperactivity disorder. Neuro. Dis. Treat. 2008; 4: 389-403.

11. Weiss, M. and Murray, C.Assessmentand management of attention-deficit hyperactivity disorder in adults. Can. Med. Assoc. 2003; 168: 715-722.
12. Faraone, S.V. and Antshel, K.M. Diagnosing and treating attention-deficit/hyperactivity disorder in adults. World Psych. 2008; 7: 131-136.

13. Heiligenstein, E., Conyers, L.M., Berns, A.R. and Miller, M.A. Preliminary normative data on DSMIV attention deficit hyperactivity disorder in college students. J. Am. Coll. Hlth. 1998; 46:185-188.

14. Weyandt, L.L., Linterman, I. and Rice, J.A. Reported prevalence of attentional difficulties in a general sample of college students. J. Psycho. Behav. Assess. 1995; 17: 293-304.

15. Kessler, R.C., Adler, L., Ames, M., et al. The World Health OrganizationaAdult ADHD Self-report scale (ASRS): A short screening scale for use in the general population. Psych. Med. 2005; 35: 245-256.

16. Shaffer, David. Attention Deficit hyperactivity disorder in adults. Am. J. Psych. 1994; 151: 633-638.

17. Kessler, R.C., Adler, L., Gruber, M.J., et al. Validity of the World Health Organization adult ADHD Self-report scale (ASRS) screener in a representative sample of health plan members. Int. J. Meth. Psych. Res. 2007; 16: 52-65. 